\title{
COMPLEMENTARY MINIMUM PRINCIPLES FOR AN ELASTIC-PLASTIC MATERIAL ${ }^{1}$
}

\author{
BY \\ H. J. GREENBERG \\ Brown University
}

1. Introduction. This paper is concerned with the mechanical behavior of bodies consisting of an isotropic elastic-plastic material which obeys the stress-strain law of Prandtl and Reuss. ${ }^{2}$ This inviscid material has a sharply defined yield point and does not exhibit work hardening. To simplify mathematical work, we shall moreover assume it to be incompressible in the elastic as well as the plastic range.

For the elastic range, Hooke's law is adopted in the form which applies to an incompressible elastic solid. In the plastic range, it is assumed that (i) the stress components satisfy the flow conditions, and (ii) the components of the plastic strain rates have to each other the same ratios as the components of the stress deviation. The stressstrain law derived from these assumptions furnishes a unique rate of change of the stress deviation when the stress and the strain rates are given.

Since the stress-strain law of Prandtl and Reuss is differential in form and is not equivalent to any finite relation between stress and strain, it is of the flow type and not the deformation type. ${ }^{3}$ As Prager $^{4}$ has pointed out, the typical boundary value problems of a plastic flow theory suppose that the state of stress is known throughout the body under consideration and prescribe either the velocities on the surface or the rates of change of the surface stresses, asking in both cases for the rates of change of the stress components in the interior. This paper is concerned with the derivation of two minimum principles relating to the mixed boundary value problem where the velocities are given on part of the surface and the rates of change of the surface stresses on the rest. For the Prandtl-Reuss theory these principles play a role analogous to that played in the theory of elasticity by the principle of Castigliano and the principle of minimum potential energy.

2. Preliminary definitions and relations. We let $\sigma_{i j}$ and $\epsilon_{i j}(i, j=1,2,3)$ denote the components of the tensors of stress and strain and define the stress deviation as

$$
s_{i j}=\sigma_{i j}-\frac{1}{3} \sigma_{k k} \delta_{i j},
$$

where the usual summation convention of the tensor calculus is used, and $\delta_{i j}$ is the Kronecker delta. On account of the assumed incompressibility of the material

$$
\epsilon_{k k}=0 .
$$

${ }^{1}$ Received May 27, 1948. The results presented in this paper were obtained in the course of research conducted under a contract sponsored jointly by the Office of Naval Research and the Bureau of Ships.

${ }^{2} \mathrm{~L}$. Prandtl, Spannungsverteilung in plastischen Körpern, Proc. 1st Internat. Congr. Appl. Mech., Delft, 1924, pp. 43-54; E. Reuss, Berücksichtigung der elastischen Formänderungen in der Plastizitätstheorie, Z. angew. Math. Mech. 10, 266-274 (1930).

${ }^{3}$ This terminology was introduced by A. A. Ilyushin in his paper Relation between the theory of Saint Venant-Lévy-Mises and the theory of small elastic plastic deformations, (Russian with English summary), Prikl. Mat. Mekh. 9, 207-218 (1946).

${ }^{4}$ W. Prager, Fundamental theorems of a new mathematical theory of plasticity, Duke Math. Journal 9, 228-233 (1942). 
In working with the equations of any flow theory, it is convenient to replace differentials by the corresponding derivatives (denoted by primes) with respect to a parameter $t$. For convenience, we speak of these derivatives as rates, although the parameter $t$ with respect to which these rates are taken need not be time. Since all equations which we write are homogeneous in these derivatives, there is no viscosity effect, and replacing the "time" $t$ by any function which increases monotonically with $t$, e. g. by an angle of twist, a torque, or a load, which increases monotonically with $t$, leaves the equations unchanged.

In the Prandtl-Reuss theory the condition for the occurrence of plastic flow is

$$
J_{2}=k^{2}
$$

where

$$
J_{2}=\frac{1}{2} s_{i j} s_{i j}
$$

denotes the second invariant of the stress deviation, and $k$ denotes the yield stress in pure shear. The sign of the quantity

$$
J_{2}^{\prime}=s_{i j} s_{i j}^{\prime}
$$

is used as the criterion for loading. Thus, $J_{2}^{\prime}>0$ indicates loading, and $J_{2}^{\prime}<0$ unloading. The stress-strain relations for this theory are

$$
\epsilon_{i j}^{\prime}=\frac{1}{2 G_{0}} s_{i j}^{\prime}+\mu s_{i j},
$$

where $G_{0}$ is the elastic shear modulus, and

$$
\mu \equiv\left\{\begin{array}{l}
0, \quad \text { wherever } J_{2}<k^{2}, \quad \text { or } J_{2}=k^{2} \quad \text { but } J_{2}^{\prime}<0, \\
\frac{s_{p q} \epsilon_{p q}^{\prime}}{2 k^{2}}, \quad \text { wherever } J_{2}=k^{2} \quad \text { and } \quad J_{2}^{\prime}=0 .
\end{array}\right.
$$

The strain rates are given in terms of the velocities $u_{i}^{\prime}$ by the equations

$$
\epsilon_{i j}^{\prime}=\frac{1}{2}\left(u_{i, i}^{\prime}+u_{i, i}^{\prime}\right)
$$

where we have assumed that the displacements $u_{i}$ and their derivatives with respect to space and $t$ are small. Physically we note that the quantity $2 k^{2} \mu$ represents the rate at which plastic work is being done. Since plastic work cannot be reclaimed, i.e. since the process of plastic deformation is irreversible, we have

$$
\mu \geq 0 \text {. }
$$

This interpretation of $\mu$ follows from (2.6), (2.7), the assumption of incompressibility and the fact that (2.6) represents a decomposition of $\epsilon_{i j}^{\prime}$ into elastic strain rate and plastic strain rate.

In the following we shall consider a body consisting of an incompressible PrandtlReuss material which occupies a volume $V$ in space bounded by a surface $S$. We shall not be concerned with the specific character of $V$ and $S$ and hence shall assume only that they are sufficiently regular so that (1) solutions exist to the problems posed and (2) the divergence theorem for the transformation of volume into surface integrals remains valid. 
We shall assume for a fixed "instant" $t$ that (1) the state of stress $\sigma_{i i}$ is known throughout the body, (2) the rate of change $T_{i}^{\prime}$ of the surface stress vector $T_{i}$ is prescribed at each point of a portion $S_{1}$ of the surface $S$, (3) the velocity $u_{i}^{\prime}$ is prescribed at each point of the remaining portion $S_{2}$ of $S$. The minimum principles we shall formulate will be for the instantaneous stress rates and strain rates throughout the interior of the body.

3. Minimum principle for the stress rates. We consider first the restrictions imposed on the stress rates $\sigma_{i j}^{\prime}$ by the conditions of equilibrium. Assuming for simplicity that there are no body forces acting, the equation ${ }^{5}$

$$
\sigma_{i j, j}^{\prime}=0
$$

must be everywhere satisfied except at most at the points of a surface $\Sigma$ separating an elastic region, $J_{2}<k^{2}$, from a plastic region, $J_{2}=k^{2}$. Across such a surface we may encounter discontinuities in individual components of $\sigma_{i j}^{\prime}$, but the stress rate vector acting across each element $d \Sigma$ of the surface must be continuous for equilibrium. Thus, if $n_{j}$ denotes the normal to $d \Sigma$, and if we denote quantities evaluated on one side of $d \Sigma$ by the superscript (1), and quantities on the other side by the superscript (2), it is not necessary that

$$
{\sigma_{i j}^{\prime(1)}}^{(1)}{\sigma_{i j}^{\prime(2)}}^{(2)} \quad i, j=1,2,3
$$

but

$$
{\sigma_{i j}^{\prime(1)}}^{(1)} n_{i}={\sigma_{i j}^{\prime(2)}}^{(2)} .
$$

must be satisfied.

As an example we note the case of torsion of a circular cylinder. Let $\tau$ denote the circumferential shearing stress and consider the boundary $r=\rho$ between the elastic and plastic portions. Then, assuming $\theta^{\prime}>0$, where $\theta$ is the angle of twist, we have $\tau^{\prime}=0$ for $r \geq \rho$, since $\tau=k$ in the plastic region, whereas in the elastic portion, $r<\rho$. Now, $\tau^{\prime}$ is a linear function of $r$, increasing from 0 at $r=0$ to a maximum as $r$ approaches $\rho$. Thus, $\tau^{\prime}$ is discontinuous as we cross the surface $r=\rho$. However, the stress transmitted across an element of $r=\rho$ is zero computed from either side, and hence continuous.

In the following, a set of stress rates $\sigma_{i j}^{\prime}$ defined throughout the body will be called a solution if it satisfies (1) the conditions of equilibrium, (2) the boundary conditions on $S_{1}$, (3) the condition that

$$
J_{2}^{\prime}=s_{i j} s_{i j}^{\prime} \leq 0
$$

throughout the plastic region $J_{2}=k^{2}$, where $s_{i j}^{\prime}$ denotes the deviation of the $\sigma_{i j}^{\prime}$, and (4) the condition that there exists a set of strain rates $\epsilon_{i j}^{\prime}$ (not necessarily unique) satisfying compatibility, incompressibility and the boundary conditions on $S_{2}$, which together with the $s_{i j}^{\prime}$ and the $s_{i j}$ satisfy the stress-strain law of Prandtl and Reuss, Eq. (2.6).

It should be noted that $\sigma_{i j}$ and hence $s_{i j}$ is known only as a function of position at the instant $t$ and so cannot, of course, simply be differentiated with respect to $t$ to yield $\sigma_{i j}^{\prime}$ and $s_{i j}^{\prime}$. However, knowledge of $s_{i j}$ as a function of position is sufficient to determine

${ }^{5} \mathrm{~A}$ subscript following a comma denotes differentiation with respect to the corresponding variable, e.g. $\sigma^{\prime}{ }_{i j, j}=\partial \sigma^{\prime}{ }_{i j} / \partial x_{j}$. 
the region $J_{2}=k^{2}$ and to verify in this region for any test solution $\sigma_{i j}^{\prime}$ whether or not $s_{i j} s_{i j}^{\prime} \leq 0$, i.e. whether (3.2) is satisfied.

Let $\sigma_{i j}^{\prime *}$ be an artificial system of stress rates, defined throughout the body, which satisfy (1) the conditions of equilibrium, (2) the boundary conditions on $S_{1}$ and (3) the condition that

$$
s_{i j} s_{i j}^{\prime *} \leq 0
$$

throughout the plastic region $J_{2}=k^{2}$, where $s_{i j}^{\prime *}$ denotes the deviation of the $\sigma_{i i}^{\prime *}$. The condition (3.3) eliminates from consideration stress rates which would act to increase $J_{2}$ in the plastic region. A system of stress rates $\sigma_{i j}^{\prime *}$ satisfying the above conditions will be called an admissible state of stress rates or simply an admissible state.

MINIMUM PRINCIPLE. If $\sigma_{i j}^{\prime}$ is a solution and $\sigma_{i j}^{\prime *}$ is an arbitrary admissible state, then

$$
I\left(\sigma_{i j}^{\prime}\right) \leq I\left(\sigma_{i j}^{\prime *}\right),
$$

where I is defined by

$$
I\left(\sigma_{i i}^{\prime *}\right)=\frac{1}{4 G_{0}} \int_{V} s_{i j}^{\prime *} s_{i j}^{\prime *} d v-\int_{S_{2}} T_{i}^{\prime *} u_{i}^{\prime} d S_{2} .
$$

Equality holds in (3.4) if and only if $s_{i j}^{\prime *}=s_{i j}^{\prime}$.

From this principle which states that $I$ is rendered an absolute minimum by a solution, it follows that if a solution $\sigma_{i j}^{\prime}$ exists, it is unique in the sense that its deviatoric part $s_{i j}^{\prime}$ is unique. This solution may be spoken of as the natural state of stress rates.

We note that this principle is not restricted in any way to loading. Indeed, under the general boundary conditions considered, unloading may conceivably occur anywhere in the body including the already plastic as well as the still elastic portions.

Proof. We first note that

$$
\int_{V} \sigma_{i j}^{\prime *} \epsilon_{i j}^{\prime} d v=\int_{S} T_{i}^{\prime *} u_{i}^{\prime} d S .
$$

where $T_{i}^{\prime *} \equiv \sigma_{i j}^{\prime *} n_{j}$ and $n_{j}$ are the components of the unit normal to $S$. This is proved in the usual manner using the equations $\sigma_{i j, i}^{\prime *}=0$ and $\epsilon_{i j}^{\prime}=1 / 2\left(u_{i, j}^{\prime}+u_{i, i}^{\prime}\right)$ and the divergence theorem to accomplish the transformation from a volume to a surface integral. Care must be taken, however, since the $\sigma_{i i}^{\prime *}$ are not necessarily continuous across a surface within the body which separates a subregion $J_{2}<k^{2}$ from a subregion $J_{2}=k^{2}$. However, the result may be proved for each subregion (assuming each is sufficiently regular) and the results added. On account of the continuity of the stress rate vector across surfaces common to two such subregions the contributions over these surfaces add to zero and we are left with the integral over $S$.

In view of the assumed incompressibility we may replace (3.6) by

$$
\int_{V} s_{i}^{\prime *} \epsilon_{i j}^{\prime} d v=\int_{S} T_{i}^{\prime *} u_{i}^{\prime} d S .
$$

Similarly, for the solution $\sigma_{i j}^{\prime}$

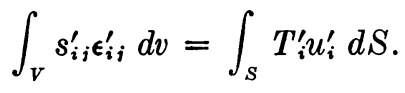


Subtracting, and substituting for $\epsilon_{i j}^{\prime}$ from (2.6), we obtain

$$
\int_{V}\left(s_{i j}^{\prime *}-s_{i j}^{\prime}\right)\left(\frac{1}{2 G_{0}} s_{i j}^{\prime}+\mu s_{i j}\right) d v=\int_{S_{2}}\left(T_{i}^{\prime *}-T_{i}^{\prime}\right) u_{i}^{\prime} d S_{2},
$$

since over $S_{1}$ we have $T_{i}^{\prime *}=T_{i}^{\prime}$. Thus,

$$
\begin{aligned}
\frac{1}{2 G_{0}} \int_{V}\left(s_{i j}^{\prime *}-s_{i j}^{\prime}\right) s_{i j}^{\prime} d v= & -\int_{V} \mu s_{i j}\left(s_{i j}^{\prime *}-s_{i j}^{\prime}\right) d v \\
& +\int_{S_{2}}\left(T_{i}^{\prime *}-T_{i}^{\prime}\right) u_{i}^{\prime} d S_{2} .
\end{aligned}
$$

From the definition of $\mu$ in (2.7), it follows that

$$
\mu s_{i} s_{i j}^{\prime}=\mu J_{2}^{\prime}=0
$$

throughout the body. Accordingly, (3.9) becomes

where

$$
\frac{1}{2 G_{0}} \int_{V} s_{i j}^{\prime} \Delta s_{i j}^{\prime} d v=-\int_{V} \mu s_{i j} s_{i j}^{\prime *} d v+\int_{S_{2}} u_{i}^{\prime} \Delta T_{i}^{\prime} d S_{2},
$$

$$
\begin{aligned}
\Delta s_{i j}^{\prime} & =s_{i j}^{\prime *}-s_{i j}^{\prime}, \\
\Delta T_{i}^{\prime} & =T_{i}^{\prime *}-T_{i}^{\prime} .
\end{aligned}
$$

Substituting from (3.12) for ${s_{i}^{\prime *}}^{\prime *}$ and $T_{i}^{\prime *}$ into (3.5) we find that

$$
\begin{gathered}
I\left(\sigma_{i j}^{\prime *}\right)=\frac{1}{4 G_{0}} \int_{V} s_{i j}^{\prime} s_{i j}^{\prime} d v-\int_{S_{2}} T_{i}^{\prime} u_{i}^{\prime} d S_{2}+\frac{1}{2 G_{0}} \int_{V} s_{i j}^{\prime} \Delta s_{i j}^{\prime} d v \\
-\int_{S_{2}} u_{i}^{\prime} \Delta T_{i}^{\prime} d S_{2}+\frac{1}{4 G_{0}} \int_{V} \Delta s_{i j}^{\prime} \Delta s_{i j}^{\prime} d v .
\end{gathered}
$$

The first two terms on the right-hand side of (3.13) constitute $I\left(\sigma_{i j}^{\prime}\right)$ and the third term can be eliminated by means of (3.11); thus,

$$
I\left(\sigma_{i j}^{\prime *}\right)-I\left(\sigma_{i j}^{\prime}\right)=-\int_{V} \mu s_{i j} s_{i j}^{\prime *} d v+\frac{1}{4 G_{0}} \int_{V} \Delta s_{i j}^{\prime} \Delta s_{i j}^{\prime} d v .
$$

The last term in (3.14) is always positive, vanishing if, and only if, $s_{i j}^{\prime *}=s_{i j}^{\prime}$. Since $\mu \geq 0$ and, by (3.13), $s_{i j} s_{i j}^{\prime *} \leq 0$ wherever $\mu>0$, it follows that the first term on the right-hand side of (3.14) is also positive or zero; this term vanishes for $s_{i j}^{\prime *}=s_{i}^{\prime}$ ince $s_{i j} s_{i j}^{\prime}=J_{2}^{\prime}=0$ for $\mu>0$. The minimum principle is thereby proved.

We remark that in the case where the stress rate vector is everywhere prescribed on the boundary, this principle reduces to the statement that the quantity

$$
\frac{1}{4 G_{0}} \int_{V} s_{i j}^{\prime *} s_{i j}^{\prime *} d v
$$

is an absolute minimum for the natural state. The integral (3.15), in effect, measures the total intensity of $s_{i j}^{\prime *}$, the non-hydrostatic and hence "plastically active" part of the stress rates $\sigma_{i j}^{\prime *}$. Thus, the principle given may be termed one of minimum stress rate intensity. 
It is interesting to compare this principle of minimum stress rate intensity with the Castigliano principle of elasticity which is one of minimum stress intensity or stress "energy". Roughly speaking, the former principle deals with minimizing the second invariant

$$
L_{2}=\frac{1}{2} s_{i j}^{\prime} s_{i j}^{\prime}
$$

of the stress rate deviation, while the latter principle for an incompressible elastic solid deals with minimizing the second invariant

$$
J_{2}=\frac{1}{2} s_{i j} s_{i j}
$$

of the stress deviation. The Castigliano principle, however, can be easily transformed into an "incremental" principle for the increments of loads and the increments of stress they produce. This incremental principle is the counterpart of the principle discussed here for plastic bodies. We note that in the elastic case the increments of stress are independent of the already existing stress, while in the plastic case they are not.

4. Minimum principle for the strain rates. We now restrict our attention to the case where the effect of the prescribed stress rates $T_{i}^{\prime}$ on $S_{1}$ and the prescribed velocities $u_{i}^{\prime}$ on $S_{2}$ turn out to be such that unloading does not occur in the plastic region, i.e. $J_{2}^{\prime}=0$ wherever $J_{2}=k^{2}$. With this restriction we prove a minimum principle for the strain rates for the Prandtl-Reuss material.

A set of strain rates $\epsilon_{i j}^{\prime}$ will be called a solution provided that it satisfies compatibility, incompressibility, the boundary conditions on $S_{2}$ and determines, together with the $\sigma_{i j}$ (which are assumed to be known as in Sec. 3), through (2.6), an equilibrium set of stress rates which satisfy the boundary conditions on $S_{1}{ }^{6}$ We assume that such a solution $\epsilon_{i j}^{\prime}$, not necessarily unique, exists. Let $\epsilon_{i j}^{\prime *}$ denote any artificial system of strain rates which satisfy compatibility, incompressibility and whose corresponding velocities $u_{i}^{\prime *}$ satisfy the boundary conditions on $S_{2}$; such a system of strain rates will be called an admissible state.

MINIMUM PRINCIPLE. If $\epsilon_{i j}^{\prime}$ is a solution and $\epsilon_{i j}^{\prime *}$ is an arbitrary admissible state, then

$$
J\left(\epsilon_{i j}^{\prime}\right) \leq J\left(\epsilon_{i j}^{\prime *}\right)
$$

where $J$ is defined by

$$
J\left(\epsilon_{i j}^{\prime *}\right)=G_{0} \int_{V}\left[\epsilon_{i j}^{\prime *} \epsilon_{i j}^{\prime *}-\mu^{*} s_{i j} \epsilon_{i j}^{\prime *}\right] d v-\int_{S_{1}} T_{i}^{\prime} u_{i}^{\prime *} d S_{1},
$$

${ }^{6}$ Actually only the stress rate deviations $s^{\prime}{ }_{i j}$ are determined by (2.6). To obtain the condition which these must satisfy for equilibrium we rewrite (3.1) in the form

$$
s_{i, j}^{\prime}-p_{, i}^{\prime}=0
$$

where $p^{\prime}=-\sigma^{\prime}{ }_{k k} / 3$ is the mean pressure rate. Eliminating $p^{\prime}$ we find that

$$
s_{i j, j k}^{\prime}-s_{k i, i i}^{\prime}=0 .
$$

Provided that the $s_{i j}^{\prime}$ satisfy this equation, we may compute the gradient of the mean pressure rate from the preceding equation. The mean pressure rate, and hence $\sigma_{i j}^{\prime}=s_{i j}^{\prime}-p^{\prime} \delta_{i j}$, is thereby determined to within a constant rate of hydrostatic pressure. It may then be checked whether the boundary conditions on $S_{1}$ are satisfied (to within a constant rate of hydrostatic pressure). 
and

$$
\mu^{*} \equiv\left\{\begin{array}{l}
0, \quad \text { wherever } J_{2}<k^{2}, \\
\frac{s_{p a} \epsilon_{p a}^{\prime *}}{2 k^{2}}, \quad \text { wherever } J_{2}=k^{2} .
\end{array}\right.
$$

Equality holds in (4.2) if and only if $\epsilon_{i j}^{\prime *}$ is a solution.

Proof. From (2.6) and (2.7) we have

where

$$
s_{i j}^{\prime}=2 G_{0}\left(\epsilon_{i j}^{\prime}-\mu s_{i i}\right)
$$

$$
\mu \equiv\left\{\begin{array}{l}
0, \quad \text { wherever } J_{2}<k^{2}, \\
\frac{s_{p q} \epsilon_{p q}^{\prime}}{2 k^{2}}, \quad \text { wherever } J_{2}=k^{2},
\end{array}\right.
$$

it having been assumed that there is no region for which $J_{2}=k^{2}$ while. $J_{2}^{\prime}<0$. We note then, by (4.3) and (4.5), that

$$
\mu^{*}=\mu \equiv 0, \quad \text { wherever } \quad J_{2}<k^{2} .
$$

From (4.4) it follows that

$$
\int_{V} s_{i j}^{\prime}\left(\epsilon_{i j}^{\prime *}-\epsilon_{i j}^{\prime}\right) d v=2 G_{0} \int_{V}\left(\epsilon_{i j}^{\prime}-\mu s_{i j}\right)\left(\epsilon_{i j}^{\prime *}-\epsilon_{i j}^{\prime}\right) d v .
$$

At the same time,

$$
\int_{V} s_{i j}^{\prime}\left(\epsilon_{i j}^{\prime *}-\epsilon_{i j}^{\prime}\right) d v=\int_{V} \sigma_{i j}^{\prime}\left(\epsilon_{i j}^{\prime *}-\epsilon_{i j}^{\prime}\right) d v=\int_{S_{1}} T_{i}^{\prime}\left(u_{i}^{\prime *}-u_{i}^{\prime}\right) d S_{1},
$$

since both $\epsilon_{i i}^{\prime *}$ and $\epsilon_{i j}^{\prime}$ satisfy compatibility and correspond to velocities $u_{i}^{\prime *}$ and $u_{i}^{\prime}$ which are identical over part $S_{2}$ of the boundary. Equating the expressions in (4.7) and (4.8) and introducing the notations

we find that

$$
\begin{aligned}
& \Delta \epsilon_{i j}^{\prime}=\epsilon_{i j}^{\prime *}-\epsilon_{i j}^{\prime} \\
& \Delta u_{i}^{\prime}=u_{i}^{\prime *}-u_{i}^{\prime}
\end{aligned}
$$

$$
2 G_{0} \int_{V} \epsilon_{i j}^{\prime} \Delta \epsilon_{i j}^{\prime} d v=2 G_{0} \int_{V} \mu s_{i j} \Delta \epsilon_{i j}^{\prime} d v+\int_{S_{1}} T_{i}^{\prime} \Delta u_{i}^{\prime} d S_{1} .
$$

Returning to (4.2), we must now substitute for $\epsilon_{i j}^{\prime *}$ and $u_{i}^{\prime *}$ from (4.9) and expand the expression obtained. We note first, that by virtuc of (4.3), (4.5), and the first equation in (4.9) we may write

where we define

$$
\mu^{\prime} s_{i j} \epsilon_{i j}^{\prime *}=\mu s_{i i} \epsilon_{i j}^{\prime}+\frac{\tau}{2 k^{2}}
$$

$$
\tau \equiv\left\{\begin{array}{l}
0, \quad \text { wherever } J_{2}<k^{2}, \\
2\left(s_{p q} \epsilon_{p q}^{\prime}\right)\left(s_{i j} \Delta \epsilon_{i j}^{\prime}\right)+\left(s_{p q} \Delta \epsilon_{p q}^{\prime}\right)\left(s_{i j} \Delta \epsilon_{i j}^{\prime}\right), \quad \text { wherever } J_{2}=k^{2} .
\end{array}\right.
$$


Carrying out the expansion of (4.2) now, using (4.9) and (4.12) we obtain

$$
\begin{aligned}
J\left(\epsilon_{i j}^{\prime *}\right)=G_{0} \int_{V} & {\left[\epsilon_{i j}^{\prime} \epsilon_{i j}^{\prime}+2 \epsilon_{i j}^{\prime} \Delta \epsilon_{i j}^{\prime}+\Delta \epsilon_{i j}^{\prime} \Delta \epsilon_{i j}^{\prime}-\mu s_{i j} \epsilon_{i j}^{\prime}\right.} \\
& \left.-\frac{\tau}{2 k^{2}}\right] d v-\int_{S_{1}} T_{i}^{\prime} u_{i}^{\prime} d S_{1}-\int_{S_{1}} T_{i}^{\prime} \Delta u_{i}^{\prime} d S_{1},
\end{aligned}
$$

or, introducing $J\left(\epsilon_{i j}^{\prime}\right)$ on the right-hand side of this equation and substituting from (4.10)

$$
J\left(\epsilon_{i j}^{\prime *}\right)-J\left(\epsilon_{i j}^{\prime}\right)=G_{0} \int_{V}\left(2 \mu s_{i j} \Delta \epsilon_{i j}^{\prime}+\Delta \epsilon_{i j}^{\prime} \Delta \epsilon_{i j}^{\prime}-\frac{\tau}{2 k^{2}}\right) d v
$$

We now examine the integrand in (4.13). Over the region $J_{2}<k^{2}$ it reduces to $\Delta \epsilon_{i j}^{\prime} \Delta \epsilon_{i j}^{\prime}$. Hence, over this region the integral in (4.13) is positive, becoming zero if, and only if, $\epsilon_{i j}^{\prime *}=\epsilon_{i j}^{\prime}$. Over the region $J_{2}=k^{2}$, we have, upon substituting for $\mu$ and $\tau$ from (4.5) and (4.12), the following expression for the integrand:

$$
\Delta \epsilon_{i j}^{\prime} \Delta \epsilon_{i j}^{\prime}-\frac{1}{2 k^{2}}\left(s_{p q} \Delta \epsilon_{p q}^{\prime}\right)\left(s_{i j} \Delta \epsilon_{i j}^{\prime}\right)=\frac{1}{2 k^{2}}\left(s_{p q} s_{p q} \Delta \epsilon_{i j}^{\prime} \Delta \epsilon_{i j}^{\prime}-s_{p q} \Delta \epsilon_{p q}^{\prime} s_{i j} \Delta \epsilon_{i j}^{\prime}\right) \geq 0,
$$

which is non-negative by Schwartz' inequality for sums. This proves (4.1) and hence that $\epsilon_{i j}^{\prime}$ renders $J$ a minimum.

It remains to consider the question of uniqueness. If in (4.1) we have equality so. that

$$
J\left(\epsilon_{i j}^{\prime *}\right)=J\left(\epsilon_{i j}^{\prime}\right),
$$

then, by what has already been said, it is necessary that

$$
\epsilon_{i j}^{\prime *} \equiv \epsilon_{i j}^{\prime}, \quad \text { in the region } J_{2}<k^{2},
$$

i.e. we have uniqueness in the elastic region. Moreover, (4.14) must reduce to an equality, which is the case if, and only if, between the qualities $s_{i j}$ and $\Delta \epsilon_{i j}^{\prime}=\epsilon_{i j}^{\prime *}-\epsilon_{i j}^{\prime}$ there holds the relation ${ }^{7}$

$$
\boldsymbol{\epsilon}_{i j}^{\prime *}-\boldsymbol{\epsilon}_{i j}^{\prime}=f s_{i j}, \quad \text { wherever } J_{2}=k^{2},
$$

where $f$ is a scalar function of position. To prove that the solution $\epsilon_{i j}^{\prime}$ is unique would be to prove that $f \equiv 0$ throughout the region $J_{2}=k^{2}$. This, the minimum principle does not tell us. Indeed, given $s_{i j}$ and $s_{i j}^{\prime}$, the stress-strain relations (4.4) do not by themselves uniquely determine the strain-rates $\epsilon_{i j}^{\prime}$. Thus, it is by no means clear that the problem has a unique solution. However, the minimum principle does provide us with the statement (4.15). From this we shall at once deduce that if $f \not \equiv 0$, then $\epsilon_{i j}^{\prime *}$ must be a solution which is distinct from $\epsilon_{i j}^{\prime}$. Hence, in any event, if for a given set of $\epsilon_{i j}^{\prime *}, J$ attains its minimum value, then $\epsilon_{i j}^{\prime *}$ is a solution. To do this, we need only show that $\epsilon_{i j}^{\prime *}$ satisfies (4.4), where $s_{i j}$ and $s_{i j}^{\prime}$ remain unchanged. Adding $f s_{i j}$ to both sides of (2.6), which is the same equation as (4.4), we have, by (4.15)

$$
\epsilon_{i j}^{\prime *}=\frac{1}{2 G_{0}} s_{i j}^{\prime}+(f+\mu) s_{i j},
$$

${ }^{7}$ The author is indebted to W. Prager for pointing out Eq. (4.15) and calling his attention to the question of the uniqueness of the strain rates. 
and we need only consider the region $J_{2}=k^{2}$. From (4.15) it follows that

so that by (4.5)

$$
f s_{i j} s_{i j}=f 2 k^{2}=s_{i j}\left(\epsilon_{i j}^{\prime *}-\epsilon_{i j}^{\prime}\right)
$$

$$
f=\frac{s_{i i} \epsilon_{i j}^{\prime *}}{2 k^{2}}-\mu .
$$

Substituting (4.17) into (4.16) completes the argument. The converse statement that, if $\epsilon_{i j}^{\prime *}$ is a solution of the problem which is distinct from $\epsilon_{i j}^{\prime}$, then $J\left(\epsilon_{i j}^{\prime *}\right)=J\left(\epsilon_{i i}^{\prime}\right)$, follows by a reversal of the above reasoning.

In the next section we shall consider the relation between the two minimum principles which have been given. However, we may notice here that the principle just proved, may be considered as a principle of minimum complementary (stress-rate) intensity. To illustrate the meaning of the term complementary we note that from (2.6) we have

$$
\epsilon_{i j}^{\prime} \epsilon_{i j}^{\prime}=\frac{1}{4 G_{0}^{2}} s_{i}^{\prime} s_{i j}^{\prime}+\frac{\dot{\mu}}{G_{0}} s_{i j}^{\prime} s_{i j}+\mu^{2} s_{i} s_{i j},
$$

and, therefore, by (2.7) and (3.10),

$$
\frac{1}{4 G_{0}} s_{i j}^{\prime} s_{i j}^{\prime}=G_{0}\left(\epsilon_{i j}^{\prime} \epsilon_{i j}^{\prime}-\mu s_{i i} \epsilon_{i j}^{\prime}\right) .
$$

On the left-hand side of (4.18) we have the stress rate intensity, on the right-hand side, the complementary quantity in the strain rates which is a minimum (under appropriate boundary conditions) by the principle of this section.

5. Relation between the principles. We find the minimum value of $I$ of (3.5) by evaluating $I\left(\sigma_{i j}^{\prime}\right)$ where $\sigma_{i j}^{\prime}$ is the natural state, i.e. the solution for the stress rates. Substituting from (4.4) into (3.5) yields

$$
I\left(\sigma_{i j}^{\prime}\right)=\frac{1}{2} \int_{V} s_{i j}^{\prime}\left(\epsilon_{i j}^{\prime}-\mu s_{i j}\right) d v-\int_{S_{2}} T_{i}^{\prime} u_{i}^{\prime} d S_{2} .
$$

Taking into account (3.8) and (3.10) we obtain

$$
I\left(\sigma_{i j}^{\prime}\right)=\frac{1}{2}\left[\int_{S_{1}} T_{i}^{\prime} u_{i}^{\prime} d S_{1}-\int_{S_{2}} T_{i}^{\prime} u_{i}^{\prime} d S_{2}\right]
$$

as the minimum value of $I$.

On the other hand, substituting from (2.6) into $J\left(\epsilon_{i j}^{\prime}\right)$ as defined by (4.2), where $\epsilon_{i j}^{\prime}$ is any solution for the strain rates, gives

$$
J\left(\epsilon_{i j}^{\prime}\right)=\frac{1}{2} \int_{V} \epsilon_{i j}^{\prime} s_{i j}^{\prime} d v-\int_{S_{1}} T_{i}^{\prime} u_{i}^{\prime} d S_{1}
$$

which, by (3.8), yields

$$
J\left(\epsilon_{i j}^{\prime}\right)=\frac{1}{2}\left[\int_{S_{2}} T_{i}^{\prime} u_{i}^{\prime} d S_{2}-\int_{S_{2}} T_{i}^{\prime} u_{i}^{\prime} d S_{1}\right]
$$

for the minimum value of the integral $J$. Comparing (5.2) and (5.4) we have the following result..$^{8}$

${ }^{8} \mathrm{Here}$ we must restrict ourselves to the case where $J_{2}^{\prime}=0$ for $J_{2}=k^{2}$, which is the one covered by the principle for the strain rates. 
For the natural state of stress rates $\sigma_{i j}^{\prime}$ and any solution $\epsilon_{i j}^{\prime}$

$$
I\left(\sigma_{i j}^{\prime}\right)=-J\left(\epsilon_{i j}^{\prime}\right)=\frac{1}{2}\left[\int_{S_{1}} T_{i}^{\prime} u_{i}^{\prime} d S_{1}-\int_{S_{2}} T_{i}^{\prime} u_{i}^{\prime} d S_{2}\right]
$$

In particular, for the case of stress rates everywhere prescribed on the boundary, i.e. $\mathrm{S}_{2}$ vacuous,

$$
\frac{1}{4 G_{0}} \int_{V} s_{i j}^{\prime} s_{i j}^{\prime} d v=G_{0} \int_{V}\left(\epsilon_{i j}^{\prime} \epsilon_{i j}^{\prime}-\mu s_{i j} \epsilon_{i j}^{\prime}\right) d v=\frac{1}{2} \int_{S} T_{i}^{\prime} u_{i}^{\prime} d S .
$$

Both (5.5) and (5.6) could, of course, be deduced directly from (4.18). Relation (5.6), in the case of elasticity, is equivalent to the familiar theorem that for the natural state, the stress energy equals the strain energy equals the work done by the external forces. The present statement, which is the one valid for elastic-plastic behavior, relates the stress rate intensity to the "work" done over the surface $S$ by the stress rate vector $T_{i}^{\prime}$ on the velocity vector $u_{i}^{\prime}$. To gain this interpretation it is more satisfactory to speak in terms of the differentials of stress and displacement, for then the integral becomes

$$
\frac{1}{2} \int_{S} d T_{i} d u_{i} d S
$$

which properly represents the work done by the $d T_{i}$ on the $d u_{i}$.

Relations (3.4), (4.1) and (5.5) can be combined to yield inequalities which furnish upper and lower bounds for the integral in (5.5). A direct method based on these inequalities of constructing approximate solutions to certain types of elastic-plastic problems will be considered in a later paper. Such methods have already been discussed in elasticity by Prager and Synge. ${ }^{9}$

6. Final remarks. In the history of the theories of plasticity various extremum principles have been formulated, of which it is relevant to mention a few. We may subdivide the various theories into theories of deformation and theories of flow (see footnote 3). The present principles appear to be the first which have been given for a theory of flow pertaining to an elastic-plastic material. Minimum principles for stress rates and strain rates for a flow theory pertaining to strain hardening materials exhibiting a continuous transition from the elastic to the plastic state were given in two papers by W. Prager. ${ }^{10}$ These are not presented as absolute minima so that strict inequalities are not given, however, in each case it is verified that the first variation vanishes and that the second variation is positive. An absolute minimum principle for the stress rates for a general flow theory of a strain hardening material has recently been given by P. Hodge and W. Prager. ${ }^{11}$ A maximum principle for the stresses for a theory of flow pertaining to a plastic material of the St. Venant-Lévy-Mises type has been given by R. Hill ${ }^{12}$ under the assumption that the body is everywhere in the plastic state.

${ }^{9}$ W. Prager and J. L. Synge, Approximations in elasticity based on the concept of function space, Quart. Appl. Math. 5, 241-269, especially 249, (1947).

${ }^{10}$ See footnote 3 and the more general, Variational principles in the theory of plasticity, to appear in the Proc. 6th Internat. Congr. Appl. Mech., Paris, 1946.

${ }^{11} \mathrm{P}$. Hodge and W. Prager, $A$ variational principle for plastic materials with strain hardening, to appear in J. Math. Phys.

${ }^{12} \mathrm{R}$. Hill, $A$ variational principle of maximum plastic work in classical plasticity, to appear in the Quart. J. Mech. Appl. Math. 
The principle of $\mathrm{A}$. Haar and Th. v. Kármán ${ }^{13}$ bears the same relationship to the deformation theory of $\mathrm{H}$. Hencky ${ }^{14}$ as the present principle of minimum stress rate intensity does to the flow theory of Prandtl and Reuss. Assuming the Haar-Kármán principle, Hencky obtained his stress-strain relations as the Euler-Lagrange equations for the integral being minimized. The Prandtl-Reuss equations can be similarly derived from the present minimum principle. Conversely, if one assumes the Hencky relations, the Haar-Kármán principle follows (this will be proved in a later note) just as we have shown the principle of minimum stress rate intensity to follow from the Prandtl-Reuss relations.

${ }^{13} \mathrm{~A}$. Haar and Th. v. Kármán, Zur Theorie der Spannungszustände in plastischen und sandartigen Medien, Nachr. Ges. Wiss. Göttingen, 204-218 (1909).

${ }^{14} \mathrm{H}$. Hencky, Über einige statische bestimmte Fälle des Gleichgewichts in plastischen Körpern, Z. angew. Math. Mech., 241-251 (1923). 